\title{
Adverse Obstetric and Perinatal Outcomes in 2333 Singleton Pregnancies Conceived After Different Endometrial Preparation Protocols: a Retrospective Study in China
}

\section{Zexin Yang}

Tianjin Medical University

\section{Xuelian Bai}

Tianjin Medical University

\section{Ying Han}

Tianjin Central Hospital of Gynecology Obstetrics

\section{Zhangxiang Zou}

Tianjin Medical University

\section{Yazhen Fan}

Tianjin Medical University

\section{Xinyan Wang}

Tianjin Central Hospital of Gynecology Obstetrics

\section{Haining Luo}

Tianjin Central Hospital of Gynecology Obstetrics

\section{Yunshan Zhang ( $\sim$ tjzys@hotmail.com )}

Tianjin Central Hospital of Gynecology Obstetrics

\section{Research Article}

Keywords: Frozen embryo transfer (FET), obstetric outcome, perinatal outcome, programmed cycle, natural cycle

Posted Date: January 20th, 2022

DOI: https://doi.org/10.21203/rs.3.rs-1141757/v1

License: (1) This work is licensed under a Creative Commons Attribution 4.0 International License. Read Full License 


\section{Abstract}

Background: Frozen-thawed embryo transfer is rising worldwide. One adverse effect of programmed frozen embryo transfer (FET) reported in some studies is an increased risk of adverse obstetric and perinatal outcomes. Meanwhile, body mass index (BMI) also has adverse effect on obstetric and perinatal outcomes. In this study, we investigated that the influence of different endometrial preparation protocols on obstetric and perinatal outcomes and the role of BMI in it.

Method: This retrospective study included 2333 singleton deliveries after frozen-thaw embryo transfer at our centre between 2014 and 2021, including 550 cycles with programmed FET, 1783 cycles with true natural cycle FET (tNC-FET). In further analysis according to BMI grouped by Asian criterion, group A $\left(18.5 \mathrm{~kg} / \mathrm{m}^{2} \leq \mathrm{BMl}<24.00 \mathrm{~kg} / \mathrm{m}^{2}\right)$ included 1257 subjects, group $B\left(24 \mathrm{~kg} / \mathrm{m}^{2} \leq \mathrm{BMK}<28.00 \mathrm{~kg} / \mathrm{m}^{2}\right)$ included 503 subjects and group $\mathrm{C}\left(\mathrm{BMI} \geq 28 \mathrm{~kg} / \mathrm{m}^{2}\right)$ included 573 subjects. Baseline characteristics of the two groups were compared and analyzed. Binary logistic regression analyses were performed to explore the association between obstetric and perinatal outcomes and endometrial preparation protocols.

Results: There were no significant differences in the placenta previa, gestational diabetes mellitus(GDM), preterm premature rupture of membranes (PPROM), cesarean section (CS) and macrosomia between the tNC-FET and programmed FET groups $(P>0.05)$. The risk of hypertensive disorders in pregnancy (HDP) was increased in programmed FET group compared with the tNC-FET group $(7.3 \%$ vs $4.4 \%$, crude OR 1.714[1.156-2.542]; adjusted OR 1.845[1.031-3.300]). After dividing the patients into three groups according to the BMI, the risk of HDP in group C (14.4\% vs 6.2\%囚crude OR 2.545 [1.424-4.548]; adjusted OR 4.712 [1.769-12.547]) was increased in programmed FET group compared with the tNC-FET group. But there was no statistically significant difference in group A and group B. Programmed FET group compared with the tNC-FET group, the risk of HDP increase as the body mass index increase.

Conclusion: This study showed a tendency toward increasing risk of HDP in programmed FET cycle compared with the INC-FET cycle, and the risk of HPD increases as BMI increases. Increased risk of preterm birth and low birth weight is linked to increased risk of HPD.

\section{Introduction}

The use of frozen-thawed embryo transfer (FET) has increased over the past decade with improvements associated with vitrification compared with older slow-freeze methods ${ }^{[1]}$. This strategy facilitates elective single-embryo transfer (e-SET), reduces the risk of ovarian hyperstimulation syndrome (OHSS), and allows time for results from preimplantation genetic testing (PGT) to return ${ }^{[2-4]}$. Moreover, potential benefits of FET include a decrease in the incidence of low birth weight, small for gestational age (SGA), preterm birth, placenta previa, placental abruption, and perinatal mortality compared with fresh embryo transfer ${ }^{[5]}$. 
However, the latest observational data assessing neonatal and maternal outcomes after FET have suggested higher rates of hypertensive disorders of pregnancy (HPD), postpartum hemorrhage, post-term birth, and macrosomia specifically in programmed FET cycles compared with natural and stimulated cycles $^{[6-9]}$.

There are three main FET protocols used in the clinic, including true natural cycle FET (NC-FET), modified natural cycle FET (mNC-FET) and programmed cycle FET (programmed FET). Cryopreserved embryos must be transferred to the uterus during the critical endometrial window to establish pregnancy ${ }^{[10]}$. For women with regular menstrual cycles, they can choose to perform in a natural cycle FET (NC-FET), whether it is a tNC-FET or a mNC-FET. In INC-FET protocol, the time of transplantation was chosen follow the natural menstrual cycle of patients. In mNC-FET protocol, ovulation is triggered by the injection of human chorionic gonadotropin ( $\mathrm{hCG}$ ) instead of a spontaneous LH surge. For women who have irregular menstrual cycles or for some logistical reasons, a programmed cycle FET (programmed FET) initiated by estradiol and progesterone is a common choice. In programmed FET protocol, the ovary is suppressed using exogenous estrogen and progesterone, and thus there is no development of a dominant follicle. Ovulation does not occur and there is no corpus luteum (CL). The time of transfer is based on the number of days that have passed since the start of exogenous progesterone.

Adverse obstetric outcomes after FET may partly be explained by the FET protocol ${ }^{[11]}$. Obstetric and perinatal outcomes after the use of different FET protocols have previously been compared. An increased risk of HDP, preterm prelabor rupture of membranes (PPROM), bleeding disorders, cesarean section (CS), post-term birth, macrosomia, and placenta accreta after programmed $\mathrm{FET}^{[12,13]}$ is observed. CL may play an important role in these in the occurrence of these complications. Recent review studies have shown that in pregnant women without $\mathrm{CL}$, the increased risk of pre-eclampsia and impaired maternal vascular health may be due to insufficient secretion of vasoactive substances, such as relaxin, vascular endothelial growth factor and so on ${ }^{[6]}$.

The purpose of our study was to investigate the obstetric and perinatal outcomes of singleton deliveries after using different FET protocols in the Chinese national cohort.

\section{Materials And Methods}

\section{Study Design}

This retrospective cohort control was conducted at the Center for Reproductive Medicine, Tianjin Central Obstetrics and Gynecology Hospital from January 1, 2014, to June 30, 2021, via our electronic records. The inclusion criteria were as follows: 1 ) age $\leq 40$ years at the time of commencement of in vitro fertilization/intracytoplasmic sperm injection (IVF/ICSI) treatment; 2 ) singleton pregnancies conceived by FET after IVF/ICSI; The following exclusion criteria were used: 1) oocyte donor treatment cycles; 2) presence of chromosomal abnormalities (included chromosome polymorphism); 3) history of uterine surgery(included endometrial polyps, cesarean and so on); 4) presence of intracavitary lesions, such as 
endometrial polyps; 5) preimplantation genetic testing; 6)slow freeze cycle. Frozen embryo transfer treatments were grouped according to the stimulation protocol used in the FET cycle, the two FET groups were defined as follows: 1) Programmed FET cycle: women who received no hCG during the FET cycle. All women were treated with progesterone and/or estradiol with or without prior downregulation with $\mathrm{GnRH}$ agonist/antagonist) tNC-FET cycle: no medication during FET cycle. We determined and grouped body mass index (BMI) as kilograms per square meter using Chinese criteria ${ }^{[14]}$. We separated the participants into three groups including the normal-weight group (group $A: 18.5 \mathrm{~kg} / \mathrm{m}^{2} \leq \mathrm{BMK}<24.00 \mathrm{~kg} / \mathrm{m}^{2}$ ), overweight group (group B: $24 \mathrm{~kg} / \mathrm{m}^{2} \leq \mathrm{BMl}<28.00 \mathrm{~kg} / \mathrm{m}^{2}$ ), and obesity group (group C: $\mathrm{BMl} \geq 28 \mathrm{~kg} / \mathrm{m}^{2}$ ). All patients underwent COS according to the routine protocol of our center.

The outcomes measured in our retrospective study were obstetric complications, including preterm birth (PTB), PPROM, HPD (including pregnancy-induced hypertension, preeclampsia, and eclampsia), placenta previa, gestational diabetes mellitus (GDM), CS, birth weight (low birth weight and macrosomia infant). PTB was defined as delivery of live birth $<37$ weeks of gestation. PPROM was defined as membranes rupture $<37$ weeks of gestation. Low-birth-weight infant was defined as birth weight $<2500 \mathrm{~g}$ and macrosomia was defined as birth weight $>4000 \mathrm{~g}$. In all groups gestational age was calculated based on a first-trimester ultrasonography scan. All diagnoses were allocated by same medical doctors. All diagnoses were allocated by medical doctors.

\section{Statistical Analysis}

Statistical analysis was performed with the use of SPSS25.0. Student t test, chi-square test, Fisher exact test, and Mann-Whitney $U$ test was used to compare the groups according to data distribution. A P value of $<0.05$ was considered to indicate statistical significance. Values are presented as mean $\pm S D$ or median (lower quartile, upper quartile) according to data distribution. Crude and adjusted odds ratios (ORs) with $95 \%$ confidence intervals (Cls) were calculated before and after adjusting for confounding variables including maternal age, infertility duration, cause of infertility, BMI, endometrial thickness and high-quality embryo transfer rate.

\section{Results}

According to the above inclusion and exclusion criteria, a total 2333 singleton deliveries after frozen-thaw embryo transfer were enrolled in our study, including 550 cycles with programmed FET, 1783 cycles with tNC-FET. In further analysis according to BMl, group A included 1257 subjects, group B included 503 subjects and group $\mathrm{C}$ included 573 subjects.

\section{Background and Treatment Characteristics}

There was no statistically significant difference in age, infertility duration, and number of embryo transfer. The mean maternal age was 31.70 and 31.49 years in the tNC-FET group and the programmed FET, respectively. Of women conceiving after $\mathrm{tNC}-\mathrm{FET}$, day 3 serum $\mathrm{FSH}$ level was significantly higher than programmed FET $(6.40 \pm 3.15$ vs $5.95 \pm 2.37, P=0.002)$. But day 3 serum LH level was significantly higher in 
programmed FET than in the tNC-FET group $(4.22 \pm 2.50$ vs $6.63 \pm 4.61, P=0.000)$. In the programmed FET group, more cycles were registered with tubal factor infertility compared with tNC-FET (36.1\% vs $24.5 \%$, $P=0.000$ ), and more cycles were registered with male factor infertility in tNC-FET group compared with programmed FET group $(16.9 \%$ vs $7.8 \%, P=0.000)$. Further, more women in the programmed FET group were diagnosed with anovulatory infertility compared with tNC-FET $(15.1 \% v s 4.0 \%, P=0.000)$. ICSI was more often used in the tNC-FET groups than in programmed FET groups ( $45.5 \%$ vs $36.5 \%, P=0.000)$, while IVF combined with ICSI was more often used in the programmed FET groups than in the tNC-FET groups (20.7\% vs $9.30 \%, P=0.000)$. Endometrial thickness of the tNC-FET group were significantly higher than in the programmed FET group (9.52 \pm 1.66 vs $9.19 \pm 1.52, P=0.002)$. All background characteristics are shown in Table 1. 
Table 1

Background and treatment characteristics of study participants according to endometrial preparation protocols

\begin{tabular}{|llll|}
\hline Parameter & tNC-FET group(n=1783) & Programmed FET group(n=550) & P \\
\hline Age (years) & $31.70 \pm 4.10$ & $30.49 \pm 3.76$ & 0.000 \\
\hline Infertility duration (years) & $4.30 \pm 2.77$ & $4.22 \pm 2.57$ & 0.570 \\
\hline BMI (kg/m2) & $23.05 \pm 3.05$ & $23.09 \pm 3.17$ & 0.538 \\
\hline Basal FSH(mIU/L) & $6.40 \pm 3.15$ & $5.95 \pm 2.37$ & 0.002 \\
\hline Basal LH(mIU/L) & $4.22 \pm 2.50$ & $6.63 \pm 4.61$ & 0.000 \\
\hline Cause of infertility, $\mathrm{n}(\%)$ & & & 0.000 \\
\hline Tubal factor & $36.1 \%(643 / 1783)$ & $24.5 \%(135 / 550)$ & 0.000 \\
\hline Anovulation & $4.0 \%(71 / 1783)$ & $15.1 \%(83 / 550)$ & 0.127 \\
\hline Diminished ovarian reserve & $1.9 \%(33 / 1783)$ & $0.9 \%(5 / 550)$ & 0.304 \\
\hline Endometriosis & $1.4 \%(25 / 1783)$ & $0.7 \%(4 / 550)$ & 0.093 \\
\hline Unexplained infertility & $4.8 \%(85 / 1783)$ & $3.1 \%(17 / 550)$ & 0.000 \\
\hline Male factor & $16.9 \%(302 / 1783)$ & $7.8 \%(43 / 550)$ & 0.000 \\
\hline Other & $35.0 \%(624 / 1783)$ & $47.8 \%(263 / 550)$ & 0.002 \\
\hline ART method, $\mathrm{n}$ (\%) & & & 0.000 \\
\hline IVF & $45.3 \%(807 / 1783)$ & $42.7 \%(235 / 550)$ & 0.000 \\
\hline ICSI & $45.5 \%(811 / 1783)$ & $36.5 \%(201 / 550)$ & 0.000 \\
\hline IVF+ICSI & $9.30 \%(165 / 1783)$ & $20.7 \%(114 / 550)$ & $9.19 \pm 1.52$ \\
\hline Endometrial thickness $(\mathrm{mm})$ & $9.52 \pm 1.66$ & $1.76 \pm 0.44$ & \\
\hline No.embroy transfer & $1.81 \pm 0.44$ & & \\
\hline
\end{tabular}

\section{Obstetric and Perinatal Outcomes after Programmed FET versus tNC-FET}

Table 2 presents the results of both the crude analyses and the analyses adjusted for maternal age, infertility duration, cause of infertility, BMI, endometrial thickness and high-quality embryo transfer rate. There were no significant differences in the placenta previa, GDM, PPROM, and macrosomia between the tNC-FET and programmed FET groups $(P>0.05)$. The risk of HDP was increased in programmed FET group compared with the tNC-FET group (7.3\% vs 4.4\%, crude OR 1.714[1.156-2.542]; adjusted OR 
1.845[1.031-3.300]). Further, a higher risk of preterm birth (9.3\% vs $12.0 \%$, crude OR 1.328[0.9811.798]; adjusted OR 1.714 [1.031-3.300]), low birth weight (5.3\% vs 8.2\%, crude OR 1.583[1.095-2.288]; adjusted OR 1.988[1.182-3.345]) was found in programmed FET pregnancies compared with tNC-FET pregnancies. CS was no longer significant in analyses after adjusting maternal age, infertility duration, cause of infertility, BMI, endometrial thickness and high-quality embryo transfer rate.

Table 2

Obstetric and perinatal outcomes after programmed FET group versus tNC-FET group

\begin{tabular}{|c|c|c|c|c|}
\hline \multirow[t]{2}{*}{ Characteristic } & \multicolumn{2}{|l|}{ Treatment } & \multicolumn{2}{|c|}{ Programmed FET vs. tNC-FET } \\
\hline & $\begin{array}{l}\text { tNC- } \\
\text { FET }(n=1783)\end{array}$ & $\begin{array}{l}\text { Programmed } \\
\text { FET }(n=550)\end{array}$ & $\begin{array}{l}\text { Crude OR (95\% } \\
\mathrm{Cl})\end{array}$ & $\begin{array}{l}\text { Adjusted OR } \\
(95 \% \mathrm{Cl})\end{array}$ \\
\hline HPD & $\begin{array}{l}4.4 \% \\
(78 / 1783)\end{array}$ & $7.3 \%(40 / 550)$ & $\begin{array}{l}1.714(1.156- \\
2.542)^{*}\end{array}$ & $\begin{array}{l}1.845(1.031- \\
3.300)^{*}\end{array}$ \\
\hline Placenta previa & $\begin{array}{l}1.0 \% \\
(17 / 1783)\end{array}$ & $0.9 \%(5 / 550)$ & $\begin{array}{l}0.953(0.350- \\
2.595)\end{array}$ & $\begin{array}{l}1.340(0.391- \\
4.586)\end{array}$ \\
\hline GDM & $\begin{array}{l}9.4 \% \\
(168 / 1783)\end{array}$ & $7.3 \%(40 / 550)$ & $\begin{array}{l}0.754(0.527- \\
1.080)\end{array}$ & $\begin{array}{l}0.901(0.557- \\
1.465)\end{array}$ \\
\hline PPROM & $\begin{array}{l}2.5 \% \\
(44 / 1783)\end{array}$ & $2.0 \%(11 / 550)$ & $\begin{array}{l}0.807(0.414- \\
1.573)\end{array}$ & $\begin{array}{l}1.119(0.464- \\
2.697)\end{array}$ \\
\hline Cesarean section & $\begin{array}{l}68.0 \% \\
(1212 / 1783)\end{array}$ & $72.9 \%(401 / 550)$ & $\begin{array}{l}1.268(1.025- \\
1.568)^{*}\end{array}$ & $\begin{array}{l}1.026(0.772- \\
1.364)\end{array}$ \\
\hline Preterm birth & $\begin{array}{l}9.3 \% \\
(166 / 1783)\end{array}$ & $12.0 \%(66 / 550)$ & $\begin{array}{l}1.328(0.981- \\
1.798)\end{array}$ & $\begin{array}{l}1.714(1.105- \\
2.659)^{*}\end{array}$ \\
\hline $\begin{array}{l}\text { Low birth weight } \\
(<2500 \mathrm{~g})\end{array}$ & $\begin{array}{l}5.3 \% \\
(95 / 1783)\end{array}$ & $8.2 \%(45 / 550)$ & $\begin{array}{l}1.583(1.095- \\
2.288)^{*}\end{array}$ & $\begin{array}{l}1.988(1.182- \\
3.345)^{*}\end{array}$ \\
\hline $\begin{array}{l}\text { Macrosomia } \\
(>4500 \mathrm{~g})\end{array}$ & $\begin{array}{l}9.9 \% \\
(177 / 1783)\end{array}$ & $11.6 \%(64 / 550)$ & $\begin{array}{l}1.195(0.882- \\
1.618)\end{array}$ & $\begin{array}{l}1.033(0.664- \\
1.605)\end{array}$ \\
\hline
\end{tabular}

\section{Subanalyses of different BMI patients on obstetric and perinatal outcomes after programmed FET versus tNC-FET}

After dividing the patients into three groups according to the BMI, the comparison results of pregnancy complications and birth weights among the three groups of patients with different endometrial preparation programs are presented in Table 3 . The risk of HDP in group C $(14.4 \%$ vs $6.2 \%$, crude OR 2.545 [1.424-4.548]; adjusted OR 4.712 [1.769-12.547]) was increased in programmed FET group compared with the tNC-FET group. But there was no statistically significant difference in group A and 
group B. Moreover, there was no an association between endometrial preparation protocols and risk of preterm birth (crude OR 1.239 [0.724-2.120]) and low birth weight (crude OR 1.932 [0.979-3.809]) in group $\mathrm{C}$ before correction for confounding factors. When the confounding variables including maternal age, infertility duration, cause of infertility, endometrial thickness and high-quality embryo transfer rate were added to the logistic regression models, the risk of preterm birth $(12.8 \%$ vs $10.6 \%$, adjusted OR 2.537 [1.164-5.527]) and low birth weight (9.1\% vs 4.9\%, adjusted OR 2.806 [1.164-5.527]) in group C was increased in programmed FET group compared with the tNC-FET group. There also was no statistically significant difference in group $A$ and group $B$. It can be seen from Figure 1 that with the body mass index increase, programmed FET group compared with the tNC-FET group, the risk of HDP, preterm birth and low birth weight also increases. There was no statistically significant difference in placenta previa, GDM, CS, PPROM and macrosomia $(P>0.05)$. 
Table 3

Subanalyses of different BMI patients on obstetric and perinatal outcomes after programmed FET versus tNC-FET

\begin{tabular}{|c|c|c|c|c|}
\hline \multirow[t]{2}{*}{ Parameter } & \multirow{2}{*}{$\begin{array}{l}\text { tNC-FET } \\
\%(n /)\end{array}$} & \multirow{2}{*}{$\begin{array}{l}\text { Programmed } \\
\text { FET } \\
\%(n /)\end{array}$} & \multicolumn{2}{|c|}{ Programmed FET vs. tNC-FET } \\
\hline & & & $\begin{array}{l}\text { Crude OR (95\% } \\
\mathrm{Cl})\end{array}$ & $\begin{array}{l}\text { Adjusted OR (95\% } \\
\mathrm{Cl})\end{array}$ \\
\hline \multicolumn{5}{|l|}{ HPD } \\
\hline$A(n=1257)$ & $\begin{array}{l}2.7 \% \\
(27 / 1010)\end{array}$ & $2.4 \%(6 / 247)$ & $\begin{array}{l}0.906(0.370- \\
2.220)\end{array}$ & $\begin{array}{l}0.703(0.189- \\
2.612)\end{array}$ \\
\hline$B(n=503)$ & $7.0 \%(27 / 387)$ & $6.0 \%(7 / 116)$ & $\begin{array}{l}0.856(0.363- \\
2.020)\end{array}$ & $\begin{array}{l}1.150(0.355- \\
3.721)\end{array}$ \\
\hline$C(n=573)$ & $6.2 \%(24 / 386)$ & $14.4 \%(27 / 187)$ & $\begin{array}{l}2.545(1.424- \\
4.548)^{\star}\end{array}$ & $\begin{array}{l}4.712(1.769- \\
12.547)^{*}\end{array}$ \\
\hline \multicolumn{5}{|c|}{ Placenta previa } \\
\hline$A(n=1257)$ & $0.9 \%(9 / 1010)$ & $1.2 \%(3 / 247)$ & $\begin{array}{l}1.367(0.367- \\
5.089)\end{array}$ & $\begin{array}{l}1.298(0.237- \\
7.102)\end{array}$ \\
\hline$B(n=503)$ & $1.3 \%(5 / 387)$ & $1.7 \%(2 / 116)$ & $\begin{array}{l}1.340(0.257- \\
7.001)\end{array}$ & - \\
\hline$C(n=573)$ & $0.8 \%(3 / 386)$ & $0.0 \%(0 / 187)$ & - & - \\
\hline \multicolumn{5}{|l|}{ GDM } \\
\hline$A(n=1257)$ & $\begin{array}{l}7.5 \% \\
(76 / 1010)\end{array}$ & $6.5 \%(16 / 247)$ & $\begin{array}{l}0.851(0.487- \\
1.487)\end{array}$ & $\begin{array}{l}1.107(0.542- \\
2.262)\end{array}$ \\
\hline$B(n=503)$ & $9.8 \%(38 / 387)$ & $6.0 \%(7 / 116)$ & $\begin{array}{l}0.590(0.256- \\
1.359)\end{array}$ & $\begin{array}{l}0.731(0.224- \\
2.387)\end{array}$ \\
\hline$C(n=573)$ & $\begin{array}{l}14.0 \% \\
(54 / 386)\end{array}$ & $9.1 \%(17 / 187)$ & $\begin{array}{l}0.615(0.346- \\
1.093)\end{array}$ & $\begin{array}{l}0.838(0.384- \\
1.832)\end{array}$ \\
\hline \multicolumn{5}{|l|}{ PPROM } \\
\hline$A(n=1257)$ & $\begin{array}{l}2.6 \% \\
(26 / 1010)\end{array}$ & $1.2 \%(3 / 247)$ & $\begin{array}{l}0.465(0.140- \\
1.550)\end{array}$ & $\begin{array}{l}0.365(0.046- \\
2.883)\end{array}$ \\
\hline$B(n=503)$ & $1.8 \%(7 / 387)$ & $1.7 \%(2 / 116)$ & $\begin{array}{l}0.952(0.195- \\
4.649)\end{array}$ & $\begin{array}{l}1.845(0.139- \\
4.410)\end{array}$ \\
\hline$C(n=573)$ & $2.8 \%(11 / 386)$ & $3.2 \%(6 / 187)$ & $\begin{array}{l}1.130(0.411- \\
3.104)\end{array}$ & $\begin{array}{l}3.345(0.743- \\
12.480)\end{array}$ \\
\hline
\end{tabular}

${ }^{*} P<0.05$ (adjusted for maternal age, infertility duration, cause of infertility, endometrial thickness and high-quality embryo transfer rate); - None data because of the small amount of subjects. 


\begin{tabular}{|c|c|c|c|c|}
\hline \multirow[t]{2}{*}{ Parameter } & \multirow{2}{*}{$\begin{array}{l}\text { tNC-FET } \\
\%(n /)\end{array}$} & \multirow{2}{*}{$\begin{array}{l}\text { Programmed } \\
\text { FET } \\
\%(n /)\end{array}$} & \multicolumn{2}{|c|}{ Programmed FET vs. tNC-FET } \\
\hline & & & $\begin{array}{l}\text { Crude OR }(95 \% \\
\text { Cl) }\end{array}$ & $\begin{array}{l}\text { Adjusted OR (95\% } \\
\text { Cl) }\end{array}$ \\
\hline$A(n=1257)$ & $\begin{array}{l}63.3 \% \\
(639 / 1010)\end{array}$ & $\begin{array}{l}64.4 \% \\
(159 / 247)\end{array}$ & $\begin{array}{l}1.049(0.785- \\
1.402)\end{array}$ & $\begin{array}{l}0.932(0.627- \\
1.384)\end{array}$ \\
\hline$B(n=503)$ & $\begin{array}{l}71.1 \% \\
(275 / 387)\end{array}$ & $80.2 \%(93 / 116)$ & $\begin{array}{l}1.647(0.992- \\
2.733)\end{array}$ & $\begin{array}{l}2.268(0.840- \\
4.748)\end{array}$ \\
\hline$C(n=573)$ & $\begin{array}{l}77.2 \% \\
(298 / 386)\end{array}$ & $\begin{array}{l}79.7 \% \\
(149 / 187)\end{array}$ & $\begin{array}{l}1.158(0.755- \\
1.777)\end{array}$ & $\begin{array}{l}0.717(0.392- \\
1.312)\end{array}$ \\
\hline \multicolumn{5}{|c|}{ Preterm birth } \\
\hline$A(n=1257)$ & $\begin{array}{l}8.5 \% \\
(86 / 1010)\end{array}$ & $11.3 \%(28 / 247)$ & $\begin{array}{l}1.374(0.875- \\
2.157)\end{array}$ & $\begin{array}{l}1.347(0.688- \\
2.639)\end{array}$ \\
\hline$B(n=503)$ & $\begin{array}{l}10.1 \% \\
(39 / 387)\end{array}$ & $12.1 \%(14 / 116)$ & $\begin{array}{l}1.225(0.640- \\
2.344)\end{array}$ & $\begin{array}{l}2.305(0.859- \\
6.181)\end{array}$ \\
\hline$C(n=573)$ & $\begin{array}{l}10.6 \% \\
(41 / 386)\end{array}$ & $12.8 \%(24 / 187)$ & $\begin{array}{l}1.239(0.724- \\
2.120)\end{array}$ & $\begin{array}{l}2.537(1.164- \\
5.527)^{*}\end{array}$ \\
\hline \multicolumn{5}{|c|}{$\begin{array}{l}\text { Low birth weight } \\
(<2500 \mathrm{~g})\end{array}$} \\
\hline$A(n=1257)$ & $\begin{array}{l}5.3 \% \\
(54 / 1010)\end{array}$ & $8.9 \%(22 / 247)$ & $\begin{array}{l}1.731(1.033- \\
2.902)^{*}\end{array}$ & $\begin{array}{l}1.467(0.688- \\
3.128)\end{array}$ \\
\hline$B(n=503)$ & $5.7 \%(22 / 387)$ & $5.2 \%(6 / 116)$ & $\begin{array}{l}0.905(0.358- \\
2.288)\end{array}$ & $\begin{array}{l}2.585(0.698- \\
9,568)\end{array}$ \\
\hline$C(n=573)$ & $4.9 \%(19 / 386)$ & $9.1 \%(17 / 187)$ & $\begin{array}{l}1.932(0.979- \\
3.809)\end{array}$ & $\begin{array}{l}2.806(1.096- \\
7.183)^{*}\end{array}$ \\
\hline \multicolumn{5}{|c|}{ Macrosomia (>4500g) } \\
\hline$A(n=1257)$ & $\begin{array}{l}8.0 \% \\
(81 / 1010)\end{array}$ & $6.1 \%(15 / 247)$ & $\begin{array}{l}0.742(0.420- \\
1.310)\end{array}$ & $\begin{array}{l}0.683(0.309- \\
1.506)\end{array}$ \\
\hline$B(n=503)$ & $\begin{array}{l}11.4 \% \\
(44 / 387)\end{array}$ & $18.1 \%(21 / 116)$ & $\begin{array}{l}1.723(0.977- \\
3.039)\end{array}$ & $\begin{array}{l}1.863(0.790- \\
4.395)\end{array}$ \\
\hline$C(n=573)$ & $\begin{array}{l}13.5 \% \\
(52 / 386)\end{array}$ & $15.0 \%(28 / 187)$ & $\begin{array}{l}1.131(0.688- \\
1.859)\end{array}$ & $\begin{array}{l}1.029(0.502- \\
2.110)\end{array}$ \\
\hline
\end{tabular}

\section{Discussion}


Our results of adverse obstetric and perinatal outcomes after procedural FET are similar to those of some recently published studies $[11-13,15,16]$. In our study we were able to adjust for maternal age, infertility duration, cause of infertility, BMI, endometrial thickness and high-quality embryo transfer rate. And to reduce the influence of confounding factors, only singletons were included. In the multiple logistic regression analysis, we illustrated that the risk of HDP, preterm birth and low birth weight was higher after FET protocols with no corpus luteum (programmed FET) compared with pregnancies after FET protocols creating a corpus luteum (tNC-FET). Moreover, grouped by the BMI of patients, programmed FET group compared with the tNC-FET group, we showed a tendency toward increasing risk of HDP, preterm birth and low birth weight as the weight of the patient increases.

A large epidemiologic study by Saito et al ${ }^{[11]}$ in Japan examined the risk for HPD in autologous pregnancies regarding the FET protocols used natural cycle $(n=29,760)$ and programmed cycle $(n=75,474)$. They determined that compared with a natural-cycle FET, pregnancies after programmed FET had increased odds of HPD (adjusted OR, 95\% Cl 1.43[1.14-1.80]). In our study, the risk of HDP (adjusted OR, 95\% Cl 1.845 [1.031-3.300]) was also increased in programmed FET group compared with the tNCFET group. Otherwise, we also showed a tendency toward increasing risk of preterm birth and low birth weight, it may be related to increased risk of HPD. After we excluded HPD in the regression analysis, the increased risk of preterm birth and low birth weight was not present (Supplementary Table 1).

Estrogen and progesterone are essential for the development of a normal placenta during pregnancy. Altered levels of these sex steroid hormones may lead to placenta-related complications in programmed FET cycle ${ }^{[17]}$. Due to failure of normal decidualization and excessive invasion of trophoblasts, low progesterone levels in early pregnancy may lead to placental implantation ${ }^{[18]}$. In contrast, some studies have shown that later development of pre-eclampsia is associated with high progesterone in early third trimester ${ }^{[19]}$. A recent overview has been shown that FET cycles have been associated with an increased risk for adverse obstetric and perinatal outcomes of a pregnancy, the absence of the CL and the following deficient circulatory adaptations during early pregnancy in programmed cycles may play a role in these increased risk ${ }^{[6]}$. The $C L$ is an important source of reproductive hormones before the establishment of the placenta as a source of pregnancy maintaining reproductive hormones, such as progesterone and estrogen. Moreover, CL also produces vasoactive products such as relaxin, vascular endothelial growth factor (VEGF), and angiogenic metabolites of estrogen ${ }^{[20]}$. They are also key factors in pregnancy maintenance. The vasoactive products produced by $\mathrm{CL}$ are thought to play an important role in early placenta formation and maternal circulation adaptation, and abnormal early placenta is often considered to be a key step in the development of preeclampsia ${ }^{[21]}$.

BMI also have important influence in IVF/ICSI outcomes. The adverse effects of overweight/obesity on pregnancy outcomes have been widely confirmed, including dysregulation of the hypothalamic-pituitaryovarian axis, ovulation disorders, impaired preimplantation embryo, and higher risk of miscarriage ${ }^{[22]}$. Meanwhile, pre-pregnancy overweight and obesity are related to many adverse obstetric and perinatal outcomes of a pregnancy, including HPD, GDM, fetal macrosomia, fetal structural anomalies, CS, preterm 
delivery and low neonatal Apgar score ${ }^{[23-26]}$. A recent study in Korean women demonstrated that prepregnancy overweight and obesity are more closely related to the adverse obstetric outcomes than excess weight gain during pregnancy ${ }^{[25]}$. In our study, considering the important impact of BMI on obstetric and perinatal outcomes of a pregnancy, we further grouped patients according to their pre-pregnancy BMI. We demonstrate that the risk of HDP in obese patients was increased in programmed FET group compared with the tNC-FET group. And a tendency toward increasing risk of HDP as the weight of the patient increases was observed. The increased risk of preterm and low birth weight has also been observed, and through further analysis we believe that it is related to the increased risk of HPD (Supplementary Table 2). PCOS/anovulation also need to be focused. In the Swedish study ${ }^{[15]}$, the investigators adjusted for anovulation, and the Chinese study included only normoovulatory women ${ }^{[12]}$ both found a higher risk of HDP. We were not able to adjust for PCOS/anovulation; however, analyses excluding all women with PCOS/anovulation did not change our results (Supplementary Table 3).

An increased risk of macrosomia in the programmed FET group was also found in the Swedish study ${ }^{[15]}$. Reasons for the increased risk of high birth weight in pregnancy after FET include maternal factors ${ }^{[27,28]}$, embryo culture media ${ }^{[29,30]}$, embryo transfer status (cleavage stage/blastocyst) ${ }^{[7,31]}$, and the quality of transferred embryos during FET. Our study also shows a tendency toward increasing risk of macrosomia. Recent study also showed that the freezing/thawing process (both slow freeze and verification) may cause epigenetic changes, which may be related to newborn weight gain ${ }^{[32,33]}$. In addition, some studies have shown that the risk of adverse obstetric and perinatal outcomes of a pregnancy such as CS increases after in the programmed FET group ${ }^{[16,34]}$. In our study, the risk of cesarean section dose not increase in the programmed FET group significantly, but the rate of CS has showed higher in programmed FET group (72.9\%) than tNC-FET group (68\%). The higher risk of adverse obstetric and perinatal outcomes after the programmed FET treatment may be responsible for the higher risk of CS.

A major strength of this study is the complete birth cohort of singletons conceived after FET in China, which minimizes the risk of selection bias. In the multiple logistic regression analysis, we were able to adjust for maternal age, infertility duration, cause of infertility, BMI, endometrial thickness and highquality embryo transfer rate. Considering the influence of BMI on obstetric and perinatal outcomes of a pregnancy, we further grouped according to BMI, firstly. Women treated with hCG trigger during FET cycle was not included in our study, because the key information absence, which is a main limitation of our study. The further study about programmed FET mNC-FET and tNC-FET should be performed in Chinese population.

\section{Conclusion}

We showed a tendency toward increasing risk of HDP in Programmed FET cycle compared with the tNCFET cycle, and the risk of HPD increases as BMI increases. Increased risk of preterm birth and low birth weight is linked to increased risk of HPD. 


\section{Abbreviations}

FET: Frozen embryo transfer; BMI: Body mass index; tNC-FET: True natural cycle FET; GDM: Gestational diabetes mellitus; PPROM: Preterm premature rupture of membranes; CS: Cesarean section; HDP: Hypertensive disorders in pregnancy; e-SET: elective single-embryo transfer; OHSS: Ovarian hyperstimulation syndrome; PGT: Preimplantation genetic testing; SGA: Small for gestational age; mNCFET: modified natural cycle; hCG: Human chorionic gonadotropin; CL: Corpus luteum; IVF/ICSI: In vitro fertilization/intracytoplasmic sperm injection; PTB: Preterm birth; OR: Odds ratios; Cl: Confidence intervals.

\section{Declarations}

\section{Ethics approval and consent to participate}

The study was approved by the Ethics Committee of the Tianjin Central Hospital of Obstetrics and Gynecology (Protocol number: ZY2021006). All participating patients were informed that their clinical data may be used for academic research in the future before entering the IVF-ET/ICSI cycle and signed written informed consents. The research was conducted ethically in accordance with the World Medical Association Declaration of Helsinki.

\section{Consent for publication}

Not applicable.

\section{Availability of data and materials}

The data used or analyzed during the current study are included within the article. The datasets are not publicly available due to the hospital policy and personal privacy. However, the datasets are available from the corresponding author on reasonable request.

\section{Competing interests}

No author has any potential conflict of interest

\section{Funding}

No funding was received.

\section{Authors' contributions}

ZXY, XLB and YSZ contributed to the study design. The analysis was made by ZXY with the assistance of $X L B$. ZXY, XLB, YH, ZXZ, YZF, XYW and HNL drafted the manuscript. ZXY and YH revised the manuscript. $Z X Y$ and $X L B$ contributed equally to this work. All authors read and approved the final manuscript. 
Acknowledgements

The authors thank all the doctors, nurses, and embryologists in the Reproductive Medicine Center of

Tianjin Central Hospital of Obstetrics and Gynecology for their help in collecting data.

\section{References}

1. RIENZI L, GRACIA C, MAGGIULLI R, et al. Oocyte, embryo and blastocyst cryopreservation in ART: systematic review and meta-analysis comparing slow-freezing versus vitrification to produce evidence for the development of global guidance [J]. Hum Reprod Update, 2017, 23(2): 139-55.

2. SAZONOVA A, KäLLEN K, THURIN-KJELLBERG A, et al. Neonatal and maternal outcomes comparing women undergoing two in vitro fertilization (IVF) singleton pregnancies and women undergoing one IVF twin pregnancy [J]. Fertil Steril, 2013, 99(3): 731-7.

3. ISHIHARA O, ARAKI R, KUWAHARA A, et al. Impact of frozen-thawed single-blastocyst transfer on maternal and neonatal outcome: an analysis of 277,042 single-embryo transfer cycles from 2008 to 2010 in Japan [J]. Fertil Steril, 2014, 101(1): 128-33.

4. MAHESHWARI A, RAJA E, BHATTACHARYA S. Obstetric and perinatal outcomes after either fresh or thawed frozen embryo transfer: an analysis of 112,432 singleton pregnancies recorded in the Human Fertilisation and Embryology Authority anonymized dataset [J]. Fertil Steril, 2016, 106(7): 1703-8.

5. SHA T, YIN X, CHENG W, et al. Pregnancy-related complications and perinatal outcomes resulting from transfer of cryopreserved versus fresh embryos in vitro fertilization: a meta-analysis [J]. Fertil Steril, 2018, 109(2): 330-42.e9.

6. SINGH B, RESCHKE L, SEGARS J, et al. Frozen-thawed embryo transfer: the potential importance of the corpus luteum in preventing obstetrical complications [J]. Fertil Steril, 2020, 113(2): 252-7.

7. ISHIHARA O, ARAKI R, KUWAHARA A, et al. Impact of frozen-thawed single-blastocyst transfer on maternal and neonatal outcome: an analysis of 277,042 single-embryo transfer cycles from 2008 to 2010 in Japan [J]. Fertil Steril, 2014, 101(1): 128-33.

8. MAHESHWARI A, PANDEY S, AMALRAJ RAJA E, et al. Is frozen embryo transfer better for mothers and babies? Can cumulative meta-analysis provide a definitive answer? [J]. Hum Reprod Update, 2018, 24(1): 35-58.

9. PINBORG A, HENNINGSEN A, LOFT A, et al. Large baby syndrome in singletons born after frozen embryo transfer (FET): is it due to maternal factors or the cryotechnique? [J]. Human reproduction (Oxford, England), 2014, 29(3): 618-27.

10. BERGH P, NAVOT D. The impact of embryonic development and endometrial maturity on the timing of implantation [J]. Fertil Steril, 1992, 58(3): 537-42.

11. SAITO K, KUWAHARA A, ISHIKAWA T, et al. Endometrial preparation methods for frozen-thawed embryo transfer are associated with altered risks of hypertensive disorders of pregnancy, placenta accreta, and gestational diabetes mellitus [J]. Human reproduction (Oxford, England), 2019, 34(8): 1567-75. 
12. JING S, LIX, ZHANG S, et al. Increased pregnancy complications following frozen-thawed embryo transfer during an artificial cycle [J]. J Assist Reprod Genet, 2019, 36(5): 925-33.

13. MAKHIJANI R, BARTELS C, GODIWALA $P$, et al. Maternal and perinatal outcomes in programmed versus natural vitrified-warmed blastocyst transfer cycles [J]. Reprod Biomed Online, 2020, 41(2): 300-8.

14. CHOO V. WHO reassesses appropriate body-mass index for Asian populations [J]. Lancet (London, England), 2002, 360(9328): 235.

15. GINSTRÖM ERNSTAD E, WENNERHOLM U, KHATIBI A, et al. Neonatal and maternal outcome after frozen embryo transfer: Increased risks in programmed cycles [J]. Am J Obstet Gynecol, 2019, 221(2): 126.e1-.e18.

16. ASSERH $\varnothing J$ L, SPANGMOSE A, AARIS HENNINGSEN A, et al. Adverse obstetric and perinatal outcomes in 1,136 singleton pregnancies conceived after programmed frozen embryo transfer (FET) compared with natural cycle FET [J]. Fertil Steril, 2021, 115(4): 947-56.

17. CHEN Z J, SHEEHAN P M, BRENNECKE S P, et al. Vessel remodelling, pregnancy hormones and extravillous trophoblast function [J]. Mol Cell Endocrinol, 2012, 349(2): 138-44.

18. JAUNIAUX E, JURKOVIC D. Placenta accreta: pathogenesis of a 20th century iatrogenic uterine disease [J]. Placenta, 2012, 33(4): 244-51.

19. TAMIMI R, LAGIOU P, VATTEN L J, et al. Pregnancy hormones, pre-eclampsia, and implications for breast cancer risk in the offspring [J]. Cancer Epidemiol Biomarkers Prev, 2003, 12(7): 647-50.

20. VON VERSEN-HöYNCK F, STRAUCH N, LIU J, et al. Effect of Mode of Conception on Maternal Serum Relaxin, Creatinine, and Sodium Concentrations in an Infertile Population [J]. Reproductive sciences (Thousand Oaks, Calif), 2019, 26(3): 412-9.

21. MILIC, NATASA, M., et al. Impaired Flow-Mediated Dilation Before, During, and After Preeclampsia A Systematic Review and Meta-Analysis [J]. Hypertension: An Official Journal of the American Heart Association, 2016,

22. BROUGHTON D, MOLEY K. Obesity and female infertility: potential mediators of obesity's impact [J]. Fertil Steril, 2017, 107(4): 840-7.

23. BRIESE V, VOIGT M, WISSER J, et al. Risks of pregnancy and birth in obese primiparous women: an analysis of German perinatal statistics [J]. Arch Gynecol Obstet, 2011, 283(2): 249-53.

24. BHATTACHARYA S, CAMPBELL D, LISTON W, et al. Effect of Body Mass Index on pregnancy outcomes in nulliparous women delivering singleton babies [J]. BMC Public Health, 2007, 7(168.

25. CHOI S, PARK I, SHIN J. The effects of pre-pregnancy body mass index and gestational weight gain on perinatal outcomes in Korean women: a retrospective cohort study [J]. Reproductive biology and endocrinology : RB\&E, 2011, 9(6.

26. JIANG L, LIN J, YAN J, et al. Prepregnancy body mass indexes are associated with perinatal outcomes in females with preeclampsia [J]. Exp Ther Med, 2020, 20(1): 500-4. 
27. EHRENBERG H M, MERCER B M, CATALANO P M. The influence of obesity and diabetes on the prevalence of macrosomia [J]. Am J Obstet Gynecol, 2004,

28. SPELLACY W N, MILLER S S, WINEGAR A, et al. Macrosomia--maternal characteristics and infant complications [J]. Obstet Gynecol, 1985, 66(2): 158-61.

29. J., C., DUMOULIN, et al. Effect of in vitro culture of human embryos on birthweight of newborns [J]. Hum Reprod, 2010, 25(3): 605-12.

30. NELISSEN E C, VAN M A P, EDITH C, et al. Further evidence that culture media affect perinatal outcome: findings after transfer of fresh and cryopreserved embryos [J]. Hum Reprod, 2012, 7): 196676.

31. ALVIGGI C, CONFORTI A, CARBONE I F, et al. Influence of cryopreservation on perinatal outcome after blastocyst- vs cleavage-stage embryo transfer: systematic review and meta-analysis [J]. Ultrasound Obstet Gynecol, 2018, 51(

32. HIURA H, HATTORI H, KOBAYASHI N, et al. Genome-wide microRNA expression profiling in placentae from frozen-thawed blastocyst transfer [J]. Clin Epigenetics, 2017, 9(1): 79.

33. GEORGIOU I, SYRROU M, PARDALIDIS N, et al. Genetic and epigenetic risks of intracytoplasmic sperm injection method [J]. Asian Journal of Andrology, 2010, 8(6): 643-73.

34. SAITO K, MIYADO K, YAMATOYA K, et al. Increased incidence of post-term delivery and Cesarean section after frozen-thawed embryo transfer during a hormone replacement cycle [J]. J Assist Reprod Genet, 2017, 34(4): 465-70.

\section{Figures}




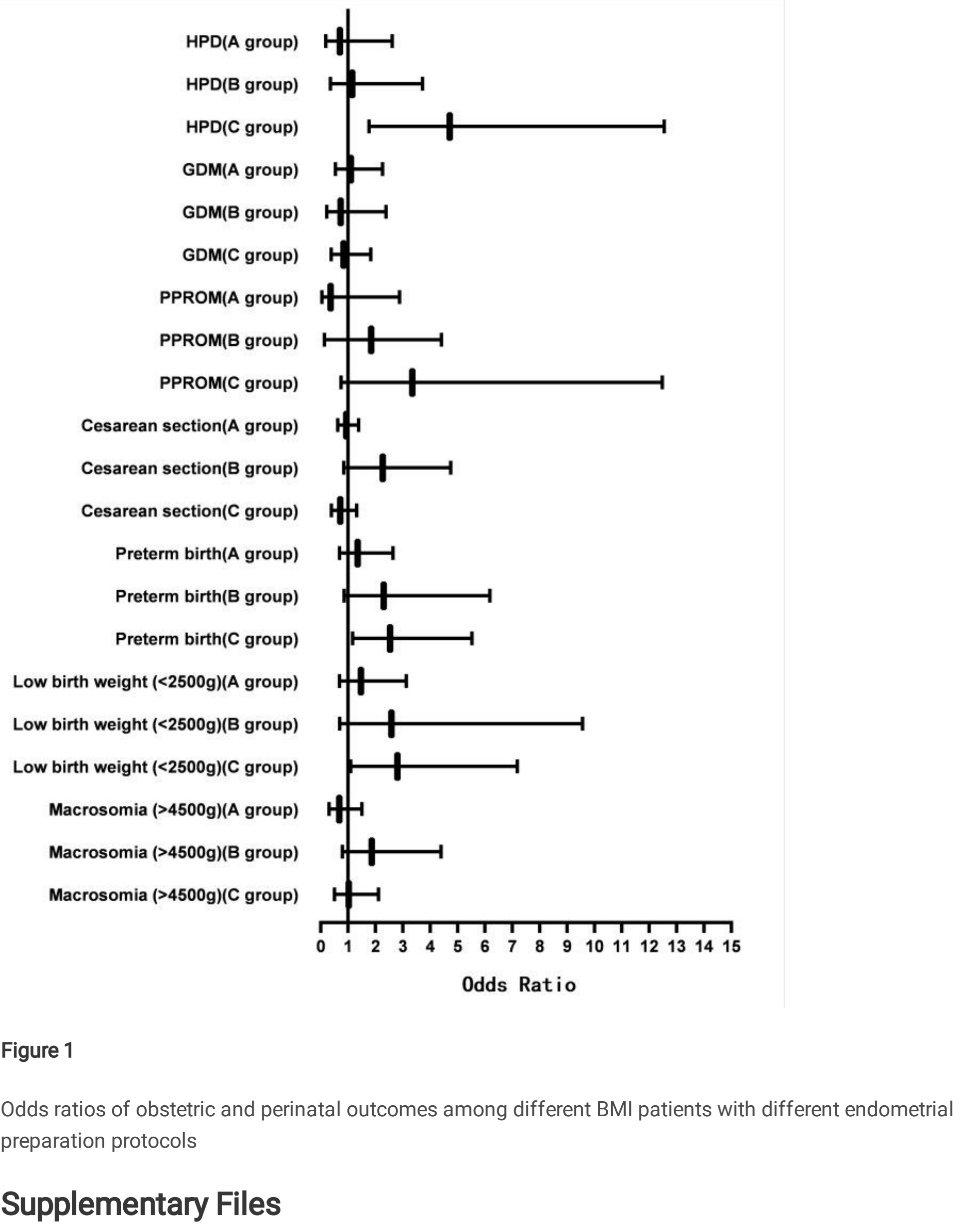


This is a list of supplementary files associated with this preprint. Click to download.

- Supplement.docx 